\title{
Prevalencia de trastornos de la articulación temporomandibular según los criterios diagnósticos para la investigación en pacientes preortodónticos
}

\author{
Prevalence of Temporomandibular Disorders based on Diagnostic \\ Research Criteria on Pre-Orthodontic Patients
}

Suly Yamily Amaya González Odontóloga, especialista en Ortodoncia, Fundación Universitaria

San Martín, Bogotá, Colombia. Docente del Posgrado de Ortodoncia, Institución Universitaria Colegios de Colombia (Unicoc), Bogotá, Colombia.

Carlos Mario Casanova Antolínez Odontólogo, especialista en Rehabilitación Oral, Pontificia Universidad Javeriana, Bogotá,

Colombia. Práctica privada.

Judith Patricia Barrera Chaparro Odontóloga, Universidad El Bosque,

Bogotá, Colombia. Especialista en Epidemiología, Universidad del

Rosario, Bogotá, Colombia. Docente en Investigación y Salud Pública, Institución Universitaria Colegios de Colombia (Unicoc), Bogotá, Colombia.

Ricardo Augusto Benavides Bonilla Odontóloga, especialista en Ortodoncia. Docente, Fundación Universitaria San Martín, Bogotá, Colombia.

Nelson Yesid Sánchez Chaparro Odontólogo, especialista en Antropología Forense, Universidad Nacional de Colombia, Bogotá, Colombia. Especialista en Ortodoncia, Fundación Universitaria San Martín,

Bogotá, Colombia. Práctica privada.

Andrea Buriticá Guarnizo Odontóloga, Universidad Nacional de Colombia, Bogotá, Colombia. Especialista en Ortodoncia, Fundación Universitaria San Martín, Bogotá, Colombia. Práctica privada.

CÓMO CITAR ESTE ARTÍCULO Amaya SY, Casanova CM, Barrera JP, Benavides RA, Sánchez NY, Buriticá A. Prevalencia de trastornos de la articulación temporomandibular según los criterios diagnósticos para bular según los criterios diagnósticos para la nvestigación en pacientes preortodónticos. Uni Odontol. 2014 Jul-Dic; 33(71): _. http://dx.doi. org/10.11144/Javeriana.uo33-71.ptat

doi:10.11144/Javeriana.uo33-71.ptat

Recibido para publicación: 08/11/2013 Aceptado para publicación: 21/09/2014

Disponible en: http://www.javeriana.edu.co/ universitasodontologica

\section{RESUMEN}

Antecedentes: es necesario evaluar la condición articular antes de iniciar el tratamiento de ortodoncia para establecer el pronóstico del tratamiento. Objetivo: determinar la prevalencia de los trastornos de la articulación temporomandibular (TTM) según los criterios diagnósticos para la investigación (CDI/TTM) en una población de pacientes preortodónticos. Métodos: estudio descriptivo de corte transversal. La muestra consistió en 158 pacientes (100 mujeres y 58 hombres), promedio de edad: $26,32 \pm$ 9,089 años, que acudieron para tratamiento ortodóntico; previa calibración teórica y clínica, tres investigadores aplicaron el instrumento CDI/TTM, que evalúa clínicamente trastornos musculares, articulares y otras alteraciones articulares (eje I) y el perfil psicosocial: dolor crónico, somatización, depresión y limitación del funcionamiento mandibular (eje II). Resultados: la prevalencia de TTM fue de $38 \%(n=60)$; los más frecuentes corresponden a los trastornos articulares $(n=55)$. El diagnóstico combinado muscular y articular se presentó en 1,3\% $(n=2)$. No se identificó diagnóstico de TMM en $62 \%$ ( $n=98$ ). El promedio de edad para los pacientes con diagnóstico de TTM fue de 28,95 años ( $\pm 10,32$ ), mayor que el promedio de edad de los que no presentaron diagnóstico con 24,71 años $( \pm 7,86)$; esta diferencia fue estadísticamente significativa $(p=0,008)$. La mayor proporción de gravedad del dolor crónico fue grado 0 $(96,8 \% ; n=153)$. Se presentó asociación estadísticamente significativa $(p=0,01)$ entre diagnóstico simple y escala de dolor crónico grado 0 . El $49,1 \%(n=27)$ de los pacientes con diagnóstico de trastorno articular reportaron limitación en el funcionamiento mandibular. Conclusión: los CDI/TTM son una herramienta útil y sencilla para identificar los TTM en pacientes preortodónticos.

\section{PALABRAS CLAVE}

diagnóstico clínico; estudios transversales; ortodoncia; prevalencia; trastornos de la articulación temporomandibular

\section{ÁREAS TEMÁTICAS}

Epidemiología oral; ortodoncia

\section{ABSTRACT}

Background: Is necessary to assess the joint condition before initiating orthodontic treatment to determine the prognosis of the treatment. Objective: To determine the prevalence of Temporomandibular Joint Disorders (TMD) according to the Research Diagnostic Criteria (RDC/TMD) in a preorthodontic patient population. Methods: A descriptive cross-sectional study was designed. The sample consisted of 158 patients (58 men and 100 women), mean age: $26.32 \pm 9.089$ years, who came for orthodontic treatment. After a theoretical and clinical calibration, three researchers applied the instrument RDC/TMD, which clinically evaluates muscle and joint disorders and/or other joint alterations (Axis I) and the psychosocial profile: chronic pain, somatization, depression, and restrained mandibular function (Axis II). Results: The prevalence of TMD was 38\% ( $n=60)$, the most common temporomandibular joint disorders correspond to $(n=55)$. The combined muscle and joint diagnosis occurred in 1.3\% ( $n=2)$. Not identified TMM diagnosis in $62 \%(n=98)$. The average age for patients diagnosed with TMD, was 28.95 years $( \pm 10.32)$, higher than the average age of diagnosis showed that 24.71 years $( \pm 7.86)$, this difference was statistically significant $(p=0.008)$. The higher proportion of chronic pain severity was grade $0(96.8 \% ; n=153)$. Presented statistically significant $(p=0.01)$ between simple diagnosis of chronic pain scale grade $0.49 .1 \%(n=27)$ of patients diagnosed with TMJ disorders reported limitation in jaw function. Conclusion: $\mathrm{RDC} / \mathrm{TMD}$ is a useful and easy-to-use tool for identifying TMD in preorthodontic patients.

\section{KEYWORDS}

clinical diagnosis; cross-sectional studies; epidemiology; orthodontics; prevalence; temporomandibular joint disorders

\section{THEMATIC FIELDS}

Buccal epidemiology; orthodontics 


\section{INTRODUCCIÓN}

Los trastornos temporomandibulares (TTM) se definen como un grupo de numerosas condiciones clínicas que involucran la musculatura masticatoria, la articulación temporomandibular y estructuras asociadas que generan dolor, limitación de los movimientos mandibulares y ruidos articulares (1-3). La prevalencia actual de los TTM varía ampliamente, debido a la falta de homogeneidad en los criterios diagnósticos adoptados por diferentes grupos de investigación. Los reportes realizados por Proffit (4) concluyen que la prevalencia de los TTM en la población general oscila entre el 5 y el $20 \%$; mientras que McNamara y colaboradores refieren dicha prevalencia en un rango del 10 al $20 \%$. Ambos utilizan el índice de Helkimo para el análisis (5). Por otra parte, Pow y colaboradores (2) y Dworkin y Leresche (6) han observado que la prevalencia en población mundial se encuentra entre el 1 y el $10 \%$.

En otros estudios epidemiológicos $(7,8)$ se ha usado el índice de disfunción clínica anamnésica y del estado oclusal propuesta por Marti Helkimo (9). Por otro lado, existen las investigaciones de Dworkin y Leresche (6) acerca de los criterios diagnósticos para la investigación (CDI) de los TTM que se aplicaron en diversos países (10-13), se han traducido a varios idiomas y constituyen una herramienta adecuada para la investigación epidemiológica sobre TTM.

En el ámbito ortodóntico se evidencia la necesidad de evaluar la condición articular antes de iniciar el tratamiento. Una condición articular alterada podría enmascarar la maloclusión de base, lo cual complicaría el diagnóstico, la evolución y el cumplimiento de los objetivos terapéuticos. De esta manera, el uso de un instrumento como el de los CDI /TTM permitiría analizar el estado de la articulación temporomandibular y lograr establecer objetivos reales de la terapéutica ortodóntica. El propósito de este estudio fue determinar la prevalencia de los TTM por medio de los CDI/TTM en una población de pacientes preortodónticos de la Clínica del Posgrado de Ortodoncia de la Fundación Universitaria San Martín (FUSM) de Bogotá, Colombia.

\section{MATERIALES Y MÉTODOS}

Los datos del presente estudio descriptivo de corte transversal se obtuvieron de 158 pacientes que consultaron para tratamiento ortodóntico en la Clínica del Posgrado de Ortodoncia de la FUSM entre septiembre del 2010 y septiembre del 2011. El estudio fue aprobado por el Comité de Ética de la Facultad de Posgrados de Odontología de la FUSM. Se incluyeron los pacientes que aceptaron participar en el estudio y firmaron un documento de consentimiento informado. Para los menores de edad se obtuvo también el consentimiento de sus padres. Se excluyeron los pacientes menores de 16 años de edad.

Para el examen clínico, tres operadores entrenados aplicaron los criterios CDI/TTM de acuerdo con las instrucciones de la versión que fuera traducida al español y validada por Dworkin y Leresche (14). La clasificación se muestra en la tabla 1.

TABLA 1

CLASIFICACIÓN DE LOS TRASTORNOS TEMPOROMANDIBULARES SEGÚN LOS CDI/TTM

\begin{tabular}{ll}
\hline & \multicolumn{1}{c}{ Eje I } \\
\hline Grupo I & A. Dolor miofacial \\
Trastornos musculares & B. Dolor miofacial con apertura limitada \\
& C. Sin diagnóstico en el grupo I \\
Grupo II Desplazamientos del disco & A. Desplazamiento del disco con reducción \\
& B. Desplazamiento del disco sin reducción, con limitación de apertura \\
& C. Desplazamientos del disco sin reducción, sin limitación de apertura \\
& D. Sin diagnóstico en el grupo II \\
Grupo III & A. Artralgia \\
Otras condiciones articulares & B. Osteoartritis \\
& C. Osteoartrosis \\
& D. Sin diagnóstico en el grupo III \\
\hline
\end{tabular}




\begin{tabular}{ll}
\hline & \multicolumn{1}{c}{ Eje II } \\
\hline 1. Grado de dolor crónico & $0-4$ \\
2. Nivel de depresión & Normal \\
& Moderado \\
& Grave \\
3. Somatización & Normal \\
& Moderado \\
& Grave \\
4. Limitación asociada al & N.o respuestas positivas /N.o de ítems respondidos \\
\hline
\end{tabular}

Se hicieron dos sesiones de calibración teórica (una para el eje I y otra para el eje II), en las que se revisó cada diagnóstico del sistema, con la asesoría de un especialista en rehabilitación oral. Luego, se realizó una prueba piloto en seis pacientes preortodónticos, quienes se evaluaron independientemente por cada operador. Se obtuvo un acuerdo del $99 \%$. Se aplicó el instrumento de recolección de la información de los $\mathrm{CDI} / \mathrm{TTM}$, que contiene una entrevista estructurada y un examen clínico. Este último fue confirmado por el observador experto. Cuando se presentaba desacuerdo en los hallazgos clínicos, se resolvía mediante una nueva evaluación clínica. La información registrada se digitó en una base de datos. Asimismo, la confiabilidad de este proceso se evaluó revisando al azar el $20 \%$ de los datos y comparando los registros originales con la información consignada en la base de datos.

Para el análisis estadístico, se describieron las variables cualitativas mediante distribuciones de frecuencia y porcentajes. Por otra parte, las variables cuantitativas se analizaron mediante medidas de tendencia central, como el promedio, y medidas de dispersión, como la desviación estándar. Se midió la variación mediante el coeficiente de variación u homogeneidad (0-10\%: homogéneo; 11-20\%: medianamente homogéneo; más del $20 \%$ : heterogéneo). Para el cálculo del tamaño de la muestra la confiabilidad fue del 95 $\%$. Para el análisis bivariado se utilizó la prueba $\chi 2$ de Pearson. Los datos fueron digitados y depurados en un archivo de Microsoft Excel ${ }^{\circledR}$ versión 2003 y se procesaron en el programa estadístico SPSS versión 15.0. Para evaluar el comportamiento normal de la muestra se utilizó el programa Minitab versión 16.0.

\section{RESULTADOS}

La muestra del estudio estuvo conformada por 158 pacientes, 58 hombres (36,7 \%) y 100 mujeres (63,3\%). El promedio de edad fue de 26,32 años ( $D E=9,089$ ), con un rango mínimo de 16 años y un máximo de 56 años, en su totalidad de raza mestiza. El nivel educativo más frecuente fue universitario (38\%), seguido de secundaria completa (36,7\%). En cuanto al ingreso económico, 53,5 \% de la muestra devengaba de 1 a 2 salarios mínimos mensuales legales vigentes (SMMLV), seguido de $22,2 \%$ con 2 a 4 SMMLV para el estándar colombiano.

En cuanto a la prevalencia de TTM, según CDI/TTM, en el eje I se diagnosticaron 60 pacientes con algún tipo de TTM, lo que indica una prevalencia de TTM del $38 \%(n=60)$ (IC 95\%: 30,32-45,63\%). De estos, los más frecuentes corresponden a trastornos articulares (grupo II) $(n=55)$. El diagnóstico combinado muscular y articular se presentó en $1,3 \%(n=2)$ de la muestra. El $62 \%(n=98)$ de los pacientes no presentó diagnóstico de TTM (tabla 2).

TABLA 2

Prevalencia de tTM Según los CDI/TtM en el EJe I

\begin{tabular}{lcc}
\hline \multicolumn{1}{c}{ Diagnóstico } & Frecuencia & Porcentaje \\
\hline TTM & 60 & 38,0 \\
$\begin{array}{l}\text { Grupo I. Trastornos } \\
\text { musculares }\end{array}$ & 3 & 1,9 \\
$\begin{array}{l}\text { Grupo II. Trastornos } \\
\text { articulares }\end{array}$ & 55 & 34,8 \\
$\begin{array}{l}\text { Combinación grupos } \\
\text { Iy II }\end{array}$ & 2 & 1,3 \\
Sin diagnóstico & 98 & 62,0 \\
\hline Total & $\mathbf{1 5 8}$ & $\mathbf{1 0 0 , 0}$ \\
\hline
\end{tabular}

En relación con la distribución de los diagnósticos de los TTM de acuerdo con los CDI/TTM en el eje I, en el grupo I (trastornos musculares) se presentaron 5 (3,2 \%) diagnósticos de dolor miofacial y 153 (96,8 \%) no presentaron diagnóstico. En el grupo II (trastornos articulares) predominó el desplazamiento discal con reducción en 29,7 \% para el lado izquierdo y 26,6 \% para el lado derecho, seguido del desplazamiento discal sin reducción con limitación de apertura derecho (1,3\%) 
e izquierdo (0,6 \%). La categoría más frecuente fue sin diagnóstico en el lado derecho $(72,2 \%)$ e izquierdo (69,6 \%). No se registró ningún diagnóstico del grupo III (otras condiciones articulares) (tabla 3).

TABLA 3

DIAGNÓSTICO DE LOS TTM DE ACUERDO CON LOS CDI/TTM EN EL EJE I

\begin{tabular}{|c|c|c|c|c|}
\hline Grupo I. Trastornos musculares & \multicolumn{2}{|c|}{ Frecuencia } & \multicolumn{2}{|c|}{ Porcentaje } \\
\hline Dolor miofacial & \multicolumn{2}{|c|}{5} & \multicolumn{2}{|c|}{3,2} \\
\hline Dolor miofacial con limitación de apertura mandibular & \multicolumn{2}{|c|}{0} & \multicolumn{2}{|c|}{0,0} \\
\hline Sin diagnóstico & \multicolumn{2}{|c|}{153} & \multicolumn{2}{|c|}{96,8} \\
\hline \multirow{2}{*}{ Grupo II. Trastornos articulares } & \multicolumn{2}{|c|}{ Derecha } & \multicolumn{2}{|c|}{ Izquierda } \\
\hline & $\mathbf{n}$ & $\%$ & $\mathbf{n}$ & $\%$ \\
\hline Desplazamiento discal con reducción & 42 & 26,6 & 47 & 29,7 \\
\hline Desplazamiento del disco sin reducción con limitación de la apertura & 2 & 1,3 & 1 & 0,6 \\
\hline Desplazamiento del disco sin reducción sin limitación de la apertura & 0 & 0,0 & 0 & 0,0 \\
\hline Sin diagnóstico & 114 & 72,2 & 110 & 69,6 \\
\hline \multirow{2}{*}{ Grupo III. Otras condiciones articulares } & \multicolumn{2}{|c|}{ Derecha } & \multicolumn{2}{|c|}{ Izquierda } \\
\hline & 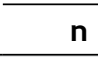 & $\%$ & $\mathbf{n}$ & $\%$ \\
\hline Artralgia & 0 & 0 & 0 & 0 \\
\hline Osteoartritis & 0 & 0 & 0 & 0 \\
\hline Osteoartrosis & 0 & 0 & 0 & 0 \\
\hline Sin diagnóstico & 158 & 100 & 158 & 100 \\
\hline
\end{tabular}

La tabla 4 muestra la distribución de los 60 diagnósticos de TTM según corresponde a diagnóstico simple o combinado. El diagnóstico simple más frecuente se presentó en el grupo II (trastornos articulares) con 55 pacientes (91,6 \%). Tres pacientes (5\%) tuvieron diagnóstico del grupo I (trastornos musculares). Se presentaron 2 pacientes (3,3\%) con diagnóstico combinado (grupos I y II) (tabla 4).

TABLA 4

DISTRIBUCIÓN DE DIAGNÓSTICOS SIMPLE Y COMBINADO DE LOS TTM SEGÚN CDI/TTM EN EL EJE I

\begin{tabular}{lrr}
\hline \multicolumn{1}{c}{ Diagnóstico de TTM } & n & \% \\
\hline Diagnóstico simple & & \\
\hline Trastornos musculares & 3 & 5,0 \\
\hline Trastornos articulares & 55 & 91,6 \\
\hline Diagnóstico doble & & \\
Trastornos musculares y articulares & 2 & 3,3 \\
(Combinación grupos I y II) & 60 & 100,0 \\
\hline
\end{tabular}

En cuanto a los diagnósticos de TTM, según los CDI/ TTM con respecto a la edad, se encontró que de los 55 diagnósticos del grupo II, 50,9 \% de los pacientes se encontraban en el rango menor o igual a 25 años. También se observó que los 3 diagnósticos del grupo I correspondían a pacientes entre 26 y 38 años.
La mayor proporción de pacientes sin diagnóstico (62,2 \%) se encontraba en el rango menor o igual a 25 años, seguido de $28,6 \%$ en pacientes de 25 a 38 años (tabla 5).

TABLA 5

DISTRIBUCIÓN DE DIAGNÓSTICOS DE TTM SEGÚN CDI/TTM CON RESPECTO A LA EDAD

\begin{tabular}{lrrrr}
\hline Edad & $\begin{array}{c}\text { I } \\
(\mathbf{n} /[\%])\end{array}$ & $\begin{array}{c}\text { II } \\
(\mathbf{n} /[\%])\end{array}$ & $\begin{array}{c}\text { I y II } \\
(\mathbf{n} /[\%])\end{array}$ & $\begin{array}{c}\text { Sin TTM } \\
(\mathbf{n} /[\%])\end{array}$ \\
\hline $\begin{array}{l}\text { Menor o } \\
\text { igual a }\end{array}$ & $0(0)$ & $28(50,9)$ & $1(50)$ & $61(62,2)$ \\
25 años & & & & \\
$\begin{array}{l}26 \text { a } 38 \\
\text { años }\end{array}$ & $3(100)$ & $16(29,1)$ & $1(50)$ & $28(28,6)$ \\
$\begin{array}{l}\text { Mayor o } \\
\text { igual a } \\
39 \text { años }\end{array}$ & $0(0)$ & $11(20)$ & $0(0)$ & $9(9,2)$ \\
$\begin{array}{l}\text { Total } \\
(158)\end{array}$ & $3(100)$ & $55(100)$ & $2(100)$ & $98(100)$ \\
\hline
\end{tabular}

El promedio de edad para los pacientes con TTM fue de 28,95 años ( $D E=10,32$ ), un poco mayor que el promedio de edad de los que no presentaron diagnóstico $(\chi 2=24,71 ; \mathrm{DE}=7,86)$. La variabilidad en cuanto a la desviación estándar fue mayor en aquellos pacientes 
que presentaron diagnóstico de TTM (figura 1). Se encontraron diferencias estadísticamente significativas entre la presencia de diagnóstico y la edad (prueba $t$ de Student, $p=0,008$ ) (tabla 6).

FIGURA 1

COMPARACIÓN DE EDAD CON PRESENCIA DE TTM SEGÚN $\mathrm{CDI} / \mathrm{TTM}^{*}$

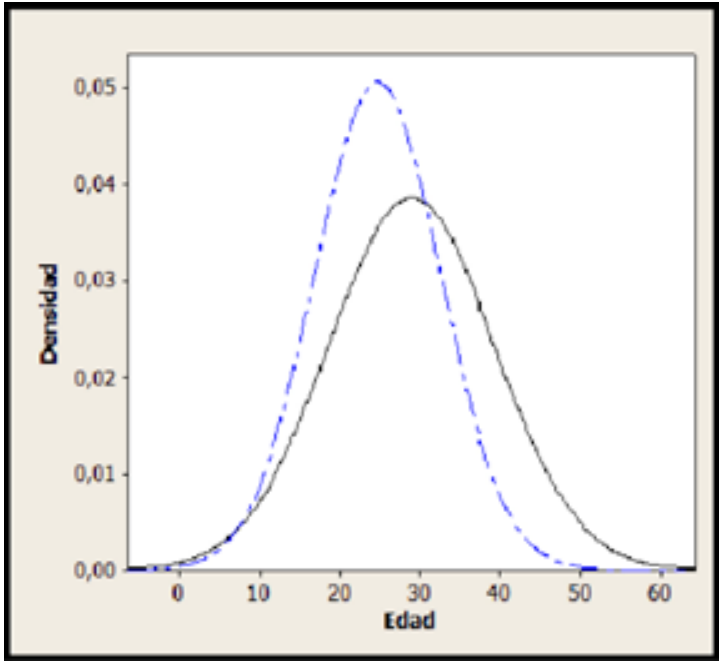

*Tipo de líneas: pacientes con diagnóstico de TTM; _ _ _ _ pacientes sin diagnóstico de TTM.
TABLA 6

COMPARACIÓN DE EDAD CON PRESENCIA DE TTM SEGÚN LOS CDI/TTM

\begin{tabular}{llll}
\hline & Media & DE & p \\
\hline Con diagnóstico de TTM & 28,95 & 10,329 & $0,008^{*}$ \\
Sin diagnóstico de TTM & 24,71 & 7,869 &
\end{tabular}

DE: desviación estándar.

*Diferencia estadísticamente significativa.

Con respecto a la distribución del perfil psicosocial, según los CDI/TTM en el eje II, se observó que para el total de la muestra la mayor gravedad de dolor crónico fue en un 96,8 \% ( $n=153)$ para pacientes del grado 0 . Ello indica ausencia de dolor o experiencia de dolor menor o igual a 6 meses. De los 60 diagnósticos de TTM, 54 pacientes con diagnóstico simple (grupo II) presentaron grado 0 en la escala de dolor crónico, lo cual fue estadísticamente significativo $(p=0,01)$.

En relación con el grado de depresión y síntomas vegetativos, todos los pacientes con diagnóstico del grupo II $(n=55)$ reportaron normalidad y solo un paciente $(33,3 \%)$ con diagnóstico del grupo I presentó un nivel moderado. La misma distribución se observó en la variable somatización con un paciente (33,3 \%) en nivel grave. En cuanto a la limitación asociada al funcionamiento mandibular, 49,1 \% ( $n=27)$ de los pacientes con diagnóstico del grupo II reportaron algún tipo de limitación. Por otra parte, 99\% ( $n=97)$ de los pacientes sin diagnóstico no reportaron limitación mandibular (tabla 7).

TABLA 7

DISTRIBUCIÓN DEL PERfiL PSICOSOCIAL SEGÚN LOS CDI/TTM EJE II CON RESPECTO A DIAGNÓSTICO DE TTM

\begin{tabular}{|c|c|c|c|c|}
\hline & I (n/[\%]) & II (n/[\%]) & $\begin{array}{c}\text { I y II } \\
\text { (n/[\%]) }\end{array}$ & $\begin{array}{c}\text { Sin TTM } \\
(\mathrm{n} /[\%])\end{array}$ \\
\hline \multicolumn{5}{|c|}{ Gravedad de dolor crónico } \\
\hline Grado 0 & $0(0)$ & $54(98,2)^{*}$ & $1(50)$ & $98(100)$ \\
\hline Grado I & $1(33,3)$ & $0(0)$ & $1(50)$ & $0(0)$ \\
\hline Grado II & $2(66,7)$ & $1(1,8)$ & $0(0)$ & $0(0)$ \\
\hline Grado III & $0(0)$ & $0(0)$ & $0(0)$ & $0(0)$ \\
\hline \multicolumn{5}{|c|}{ Nivel de depresión y síntomas vegetativos } \\
\hline Normal & $2(66,7)$ & $55(100)$ & $2(100)$ & $98(100)$ \\
\hline Moderado & $1(33,3)$ & $0(0)$ & $0(0)$ & $0(0)$ \\
\hline Grave & $0(0)$ & $0(0)$ & $0(0)$ & $0(0)$ \\
\hline \multicolumn{5}{|c|}{ Escala de síntomas físicos no específicos (somatización) } \\
\hline Normal & $2(66,7)$ & $55(100)$ & $2(100)$ & $98(100)$ \\
\hline Moderado & $0(0)$ & $0(0)$ & $0(0)$ & $0(0)$ \\
\hline Grave & $1(33,3)$ & $0(0)$ & $0(0)$ & $0(0)$ \\
\hline
\end{tabular}




\begin{tabular}{lrrrr}
\hline & I (n/[\%]) & II (n/[\%]) & $\begin{array}{c}\text { Iy II } \\
(\mathbf{n} /[\%])\end{array}$ & $\begin{array}{r}\text { Sin TTM } \\
(\mathbf{n} /[\%])\end{array}$ \\
\hline Limitación asociada al funcionamiento mandibular & & & & \\
Sí & $2(66,7)$ & $27(49,1)$ & $2(100)$ & $1(1)$ \\
No & $1(33,3)$ & $28(50,9)$ & $0(0)$ & $97(99)$ \\
\hline${ }^{*} p=0,05$, estadísticamente significativo. & & & &
\end{tabular}

\section{DISCUSIÓN}

La complejidad diagnóstica de los TTM, independientemente de cuál sea el trastorno funcional de los componentes del sistema masticatorio, hace difícil establecer el factor etiológico primario de la maloclusión. No obstante, sin tener en cuenta el sistema utilizado para clasificarlos, la identificación temprana de los TTM es uno de los aspectos relevantes para desarrollar un adecuado diagnóstico y terapia en ortodoncia. Se conoce la estrecha relación entre la presencia de maloclusión y un estado articular alterado (15), porque con mucha frecuencia involucra el componente neuromuscular. Ello deriva en un cambio en la posición mandibular con respecto a la relación céntrica. Este hecho hace que lograr diagnósticos ortodónticos y articulares eficaces y oportunos sea prioritario, con el fin de facilitar el cumplimiento de objetivos terapéuticos integrales y contribuir a la estabilidad del sistema estomatognático. Sin duda alguna, el desconocimiento de la posición y condición articular previa a un tratamiento ortodóntico puede conducir a diagnósticos errados, producto de condiciones interoclusales adaptativas que alejan la posición de relación céntrica del cóndilo con la eminencia articular. Ello da como resultado una condición oclusal habitual que afecta el resultado del diagnóstico y su tratamiento. Los CDI/TTM empleados en la presente investigación se han utilizado en estudios recientes en países como Alemania (Schmitter y colaboradores [16]), México (Cassanova-Rossado y colaboradores [17]), Italia (Manfredini y colaboradores [10]) y Chile (Vargas y Díaz [11]). Estos criterios constituyen un sistema diagnóstico cada vez más aceptado y adoptado como instrumento para medir los signos y síntomas de los TTM (18).

El presente estudio descriptivo se realizó en un grupo de población colombiana con un rango de edad entre 16 y 56 años, que asistía a la clínica de los Posgrados en Odontología de la FUSM, sede Bogotá, para tratamiento ortodóntico, y cuyo principal motivo de consulta no fuera una alteración de tipo articular. Se observó que la prevalencia de TTM fue del $38 \%$, similar a Vargas y Díaz (11), quienes informan de una prevalencia del $36,5 \%$, así como Schmitter y colaboradores (16), para quienes la prevalencia fue del $35 \%$ en pacientes de 25 a 60 años de edad. Esta prevalencia se considera alta si se compara con otros estudios como el de Yap y colaboradores (19), en los que se aplicaron los CDI/TTM en pacientes con edades entre 23 y 43 años con una condición articular preexistente. Es diferente a lo detectado por Manfredini y colaboradores (10), cuyo estudio mostró una prevalencia de TTM del 81,4 \% en pacientes con edades de 18 a 80 años. Por otro lado, el porcentaje de pacientes hallados sin diagnóstico (62 \%) parece ser representativo; pero, dadas las características mencionadas de la población del presente estudio, se esperaba un porcentaje mayor. Por este motivo, al no contar con estudios en poblaciones similares, estos resultados justifican una mayor atención a la presencia de TTM independientemente del motivo de consulta.

Del total de la muestra, la mayor frecuencia de pacientes con diagnóstico de TTM estuvo representada en el grupo II (trastornos articulares) con 34,8 \%, y dentro de este grupo el desplazamiento discal con reducción del lado izquierdo tuvo el mayor porcentaje $(29,7 \%)$. Otros autores $(6,20)$ han reportado que este diagnóstico oscila entre $10 \%$ y $34 \%$ para cada articulación, por lo que no hay una mayor diferencia con esta investigación. Por otra parte, el desplazamiento discal sin reducción del lado derecho con limitación de la apertura se presentó en un 1,3\%. Este último fue el de menor prevalencia, igual como lo sugieren otros estudios (1). La baja frecuencia encontrada para este diagnóstico se explicaría por la mayor dificultad que el disco tiene de regresar a su posición natural, si se tiene en cuenta que no es un estado crónico como el desplazamiento con reducción.

Los diagnósticos de artralgia, osteoartritis y osteoartrosis, correspondientes al grupo III de los CDI/TTM, no se presentaron en este estudio. Esto contrasta con prevalencias del $14-50 \%$ en otras publicaciones $(6,10)$. Estas diferencias pueden interpretarse por la media de edad más alta de dichos estudios (18 a 80 años) y el motivo de consulta por su condición articular. 
Otro hallazgo interesante del presente estudio tiene que ver con la baja prevalencia de diagnósticos múltiples (3,3\%), lo cual sugiere una menor complejidad diagnóstica y clínica, en contraste con el $64 \%$ reportado en población italiana (10). Si bien se esperaría que la cantidad de diagnósticos combinados aumentara en proporción a la edad, los estudios de Manfredini y colaboradores $(10,21)$ reportan su mayor prevalencia en menores de 25 años. Se asumirá, entonces, que la menor prevalencia de diagnósticos múltiples para el presente estudio se podría explicar por la baja percepción de la condición articular del paciente, debido a la ausencia de sintomatología dolorosa en los componentes del sistema masticatorio y su mayor capacidad adaptativa, tolerancia fisiológica y cronicidad.

El promedio de edad para los pacientes con TTM fue de 28,95 años ( $D E=10,32$ ) mayor que el promedio de edad de los que no presentaron diagnóstico $(\chi 2=$ 24,71 ; $D E=7,86$ ). La variabilidad fue mayor en aquellos pacientes que presentaron diagnóstico de TTM, entre quienes se hallaron diferencias estadísticamente significativas que concuerdan con lo reportado por Manfredini y colaboradores (10). Esto podría interpretarse como una tendencia a aumentar la prevalencia general por aumento de la edad poblacional.

Los CDI/TTM son un instrumento sencillo diseñado para optimizar la confiabilidad de los hallazgos en entornos de investigación y así reducir la variabilidad de los métodos de control y juicio clínico $(7,22,23)$. Sin embargo, este sistema es limitado en cuanto al número de diagnósticos y clasificaciones que incluye. Debido a que es un método simplificado para la identificación de los TTM, se descartan algunas entidades descritas en otras clasificaciones de TTM.

En este estudio, los investigadores no recibieron entrenamiento por parte de un examinador del consorcio CDI/TTM. No obstante, se contó con la supervisión de un clínico con años de experiencia en el estudio y manejo de TTM, estrategia que se ha reportado en otros estudios (10). Igualmente, esto no afectó la confiabilidad de los datos, ya que cada hallazgo clínico fue verificado por el clínico experto. Por lo tanto, este sistema de diagnóstico mostró ser aplicable por profesionales de especialidades diferentes a la rehabilitación oral y la cirugía maxilofacial, en este caso, estudiantes de Ortodoncia entrenados.

Por otra parte, el eje II de los CDI/TTM permite evaluar el perfil psicosocial del paciente mediante escalas de gravedad del dolor crónico, depresión, somatización y limitación del funcionamiento mandibular para correlacionarlo con el tipo de alteración articular. Son escasos los estudios que reportan resultados del eje II $(12,21,24)$. En el presente estudio los pacientes diagnosticados con TTM del grupo II (trastornos articulares) presentaron grado 0 , en cuanto a gravedad de dolor crónico, es decir, no presentaban dolor. Esta asociación fue estadísticamente significativa. El mismo grupo presentó grados normales con respecto a depresión y somatización. Asimismo, la mitad de dichos pacientes reportó limitación del funcionamiento mandibular. Por lo tanto, se encontró que para la población evaluada la presencia de una alteración articular del grupo II no estaba asociada a dolor ni incapacidad, ya que no era percibida por los pacientes, independientemente de la limitación del funcionamiento mandibular. Estos hallazgos son concordantes con Espinosa y colaboradores (12) y Wright y colaboradores (25), quienes hallaron que los individuos con diagnóstico simple presentaban los más bajos niveles de dolor crónico, depresión y somatización. En otros estudios, en población que consultó por problemas articulares, los grados de dolor crónico se asociaron con los TTM y las características psicosociales de los pacientes $(6,12,26)$. Por ende, a mayor disfunción psicosocial, mayor el riesgo de presentar dolor crónico en los pacientes con TTM (12).

En cuanto a los trastornos musculares, algunos autores $(6,12,27)$ han concluido que los pacientes con trastornos miogénicos presentan puntuaciones elevadas en su perfil psicosocial y muestran una correlación positiva entre la gravedad del dolor crónico y altos índices de depresión y somatización. Esto se observó en el presente estudio, en el que de los tres pacientes del grupo I (trastornos musculares) diagnosticados con TTM, una paciente mujer reportó grado II en gravedad de dolor crónico, grado moderado de depresión y grado grave de somatización.

La implementación del instrumento CDI/TTM del eje II en población latinoamericana presenta limitaciones, por las diferencias socioculturales en la percepción e impacto de los TTM en los aspectos laboral, social y cotidiano. Ello pudo influir en la presente investigación, si se tiene en cuenta que la mayoría de las poblaciones en las que se han aplicado los CDI/ TTM son de origen norteamericano y europeo. En este estudio fue necesario modificar algunos términos de la versión traducida al español, debido a que no eran comprensibles para los pacientes o no eran aplicables al contexto colombiano. De esta manera se

.


minimizaron las limitaciones del instrumento, el cual reveló que la población estudiada presentaba grados muy bajos de gravedad de dolor crónico, depresión y somatización, aspectos que pueden ser asunto de estudios posteriores.

\section{CONCLUSIONES}

De acuerdo con los resultados del presente estudio, se concluye que la prevalencia de TTM, según los CDI/TTM, fue del $38 \%$; los diagnósticos de TTM más frecuentes (34,8\%) correspondieron a trastornos articulares; se presentó asociación significativa entre pacientes menores de 25 años y ausencia de TTM, y hubo asociación significativa entre la presencia de diagnóstico articular y la gravedad de dolor crónico grado 0 .

\section{RECOMENDACIONES}

Se recomienda la aplicación de los CDI/TTM en la práctica clínica del ortodoncista como herramienta para el tamizaje de TTM, de manera que se clasifique a los pacientes para que los casos positivos sean derivados a las etapas subsecuentes de diagnóstico. Asimismo, se sugiere realizar estudios epidemiológicos que permitan comparar la prevalencia de TTM posterior a los tratamientos de ortodoncia y rehabilitación oral, así como evaluar otras poblaciones de pacientes preortodónticos, con el fin de formular protocolos de manejo que sean eficientes en la práctica clínica.

\section{AGRADECIMIENTOS}

Los autores agradecen al profesor Édgar lbáñez, por su asesoría y por el procesamiento estadístico de los datos del estudio.

\section{REFERENCIAS}

1. Sessle BJ. Evolution of the research diagnostic criteria for temporomandibular disorders. J Orofac Pain. 2010 Winter; 24(1): 5-22.

2. Pow EH, Leung KC, McMillan AS. Prevalence of symptoms associated with temporomandibular disorders in Hong Kong Chinese. J Orofac Pain. 2001 Summer; 15(3): 228-34.

3. Km MR, Graber TM, Viana MA. Orthodontics and temporomandibular disorder: a meta-analysis. Am J Orthod Dentofacial Orthop. 2002 May; 121(5): 438-46.

4. McNamara JA Jr, Seligman DA, Okeson JP. Occlusion, orthodontic treatment, and temporomandibular disorders: a review. J Orofac Pain. 1995 Winter; 9(1): 73-90.
5. Okeson JP. Current terminology and diagnostic classification schemes. Oral Surg Oral Med Oral Pathol Oral Radiol Endod. 1997 Jan; 83(1): 61-4.

6. Dworkin S, Leresche L. Research diagnostic criteria for temporomandibular disorders: review, criteria, examinations and specifications, critique. J Craniomand Dis. 1992; 6(4): 301-55.

7. Thilander B, Rubio G, Pena L, De Mayorga C. Prevalence of temporomandibular dysfunction and its association with malocclusion in children and adolescents: an epidemiologic study related to specified stages of dental development. Angle Orthod. 2002 Apr; 72(2): 146-54.

8. Ministerio de Salud, República de Colombia. III Estudio nacional de salud bucal, ENSAB III. Estudio nacional de factores de riesgo de enfermedades crónicas, ENFREC II. Tomo VII: Estudio nacional de salud bucal. Bogotá: Ministerio de Salud; 1999.

9. Helkimo M. Studies on function and dysfunction of the masticatory system. II. Index for anamnestic and clinical dysfunction and occlusal state. Sven Tandlak Tidskr. 1974 Mar; 67(2): 101-21.

10. Manfredini D, Piccotti F, Ferronato G, Guarda-Nardinl L. Age peaks of different RDC/TMD diagnoses in a patient population. J Dent. 2010 May; 38(5): 392-9.

11. Vargas P, Díaz W. Prevalencia de trastornos temporomandibulares según criterios diagnósticos para la investigación en un grupo de adultos de Santiago de Chile. Rev Dental de Chile. 2006; 423-50.

12. Espinosa I, Lara A. Comparación de los aspectos psicosociales (eje II) de los pacientes con trastornos temporomandibulares, de acuerdo a la combinación de diagnósticos físicos (eje l) de los criterios diagnósticos para la investigación de los trastornos temporomandibulares (CDI/TTM). Rev Oral. 2009; 8: 447-81.

13. Manfredini D, Chiapege, G. Research diagnostic criteria for temporomandibular (RCD/TMD) axis I diagnoses in an Italian patient population. J Oral Rehab. 2006; 33(8): 551-8.

14. Dworkin S, Leresche L. Research diagnostic criteria for temporomandibular disorders. J Craniomand Dis linternet]. 2002. [Citado 1 feb 2010]. Disponible en: http:// www.rdc-tmdinternational.org/Portals/18/Translations_RDC/RDC-Spanish.pdf.

15. Goldstein BH. Temporomandibular disorders: a review of current understanding. Oral Surg Oral Med Oral Pathol Oral Radiol Endod. 1999 Oct; 88(4): 379-85.

16. Schmitter M, Rammelsberg P, Hassel, A. The prevalence of signs and symptoms of temporomandibular disorders in very old subjects. J Oral Rehab. 2005 Jul; 32(7): 467-73.

17. Casanova-Rosado JF, Medina-Solís CE, Vallejos-Sánchez EA, Casanova-Rosado AJ, Hernández-Prado B, Avila-Burgos L. Prevalence and associated factors for temporomandibular disorders in a group of Mexican adolescents and youth adults. Clin Oral Investig. 2006 Mar; 10(1): 42-9. 
18. Quijada C, Larrucea C, Castro R, Rodríguez C. Prevalencia de trastornos temporomandibulares y relación con stress emocional en patologías neurológicas de base. Rev Fac de Odontol Univ Valparaíso. 2007; 3(5): 5-92.

19. Yap AU, Dworkin SF, Chua EK, List T, Tan KB, Tan HH. Prevalence of temporomandibular disorder subtypes, psychologic distress, and psychosocial dysfunction in Asian patients. J Orofac Pain. 2003 Winter; 17(1): 21-8.

20. List T, Dworkin S. Comparing TMD diagnoses and clinical findings at Swedish and US TMD centers using research diagnostic criteria for temporomandibular disorders. J Orofac Pain. 1996 Summer; 10(3): 240-53.

21. Manfredini D, Winocur E, Ahlberg J, Guarda-Nardini L, Lobbezoo F. Psychosocial impairment in temporomandibular disorders patients. RDC/TMD axis II findings from a multicentre study. J Dent. 2010 Oct; 38(10): 765-72.

22. Zielinsky L. Nueve tests de Krogh-Paulsen para determinar la existencia de disfunción del sistema estomatognático. Rev Ateneo Arg Odontol. 1982; 26(1): 12-78.

23. Okeson JP. Current terminology and diagnostic classification schemes. Oral Surg Oral Med Oral Pathol Oral Radiol Endod.1997 Jan; 83(1): 61-4.

24. Kanan A, Guarneros A, Espinosa I, Lara M, Vargas H. Características psicosociales de los pacientes con trastornos temporomandibulares miogénicos. Oral. 2011 Junio; 12(37): 698-701.

25. Wright AR, Gatchel RJ, Wildenstein L, Riggs R, Buschang $P$, Ellis E. Biopsychosocial differences between high-risk and low-risk patients with acute TMD-related pain. J Am Dent Assoc. 2004 Apr; 135(4): 474-83.

26. Turner JA, Mancl L, Aaron LA. Short- and long-term efficacy of brief cognitive-behavioral therapy for patients with chronic temporomandibular disorder pain: a randomized, controlled trial. Pain. 2006 Apr; 121(3): 181-94.

27. Suvinen TI, Hanes KR, Gerschman JA, Reade PC. Psychophysical subtypes of temporomandibular disorders. J Orofac Pain. 1997 Summer; 11(3): 200-5.

\section{CORRESPONDENCIA}

Suly Yamily Amaya González

sulyamgo@yahoo.es

Carlos Mario Casanova Antolínez

camanova@hotmail.com

Judith Patricia Barrera Chaparro

barrerajudith@gmail.com

Ricardo Augusto Benavides Bonilla

ricardoabenavides@gmail.com

Nelson Yesid Sánchez Chaparro

nysanchezc@gmail.com
Andrea Buriticá Guarnizo

andrea_buritica@hotmail.com 
